\title{
Evaluation of Shape-Based Normalization in the Corpus Callosum for White Matter Connectivity Analysis
}

\author{
Hui Sun ${ }^{1}$, Paul A. Yushkevich ${ }^{1}$, Hui Zhang ${ }^{1}$, Philip A. Cook ${ }^{1}$, Jeffrey T. Duda ${ }^{1}$, \\ Tony J. Simon ${ }^{2}$, and James C. Gee ${ }^{1}$ \\ ${ }^{1}$ Penn Image Computing and Science Laboratory, Department of Radiology, University of \\ Pennsylvania, Philadelphia, USA \\ ${ }^{2}$ Cognitive Analysis and Brain Imaging Laboratory, M.I.N.D. Institute, University of \\ California, Davis, USA
}

\begin{abstract}
Recently, concerns have been raised that the correspondences computed by volumetric registration within homogeneous structures are primarily driven by regularization priors that differ among algorithms. This paper explores the correspondence based on geometric models for one of those structures, midsagittal section of the corpus callosum (MSCC), and compared the result with registration paradigms. We use geometric model called continuous medial representation (cm-rep) to normalize anatomical structures on the basis of medial geometry, and use features derived from diffusion tensor tractography for validation. We show that shape-based normalization aligns subregions of the MSCC, defined by connectivity, more accurately than normalization based on volumetric registration. Furthermore, shape-based normalization helps increase the statistical power of group analysis in an experiment where features derived from diffusion tensor tractography are compared between two cohorts. These results suggest that $\mathrm{cm}$-rep is an appropriate tool for normalizing the MSCC in white matter studies.
\end{abstract}

\section{Introduction}

Group analysis of the "appearance" or "texture" features in medical images requires establishing correspondence between individual images. The problem of correspondence can be stated in terms of "normalization": to remove the anatomical differences between individuals so that group comparison can be carried out. This is usually achieved by volumetric registration, which warps the individual image into the template's space. Volumetric registration is usually driven by two terms: the similarity metric and the regularity prior. Many regularization priors (elastic, fluid, diffeomorphic et. al) are usually used to enforce the smoothness of the map between images. Within homogeneous structures, because of the lack of intensity information, the correspondences computed by the volumetric registration could be primarily driven by regularization priors that differ among algorithms [12]. In these cases, geometric models can provide a basis for normalization, especially given the large body of research on correspondence methods for these models [6, 8]. Validation of the normalization is usually hard, due to the difficulty to obtain gold standard. In this paper, validation is performed with the help of Diffusion Tensor MRI (DTI), where the alignment of the "hidden" features obtained from diffusion tensor tractography are evaluated after normalization. 
This paper is concerned with a particular type of geometric model: the continuous medial representation (cm-rep) [15]. Medial representations (m-rep) [11] describe structures by explicitly defining the medial axis (skeleton) of the structure and deriving boundary geometry from the medial axis. The PDE-based cm-rep approach [15] takes particular care to ensure that the precise geometric relationship between the medial axis and the derived boundary is maintained. 3D cm-rep models have been successfully built and applied in neuroimage studies [16]. In particular, a closed-form solution is derived for the $2 \mathrm{D}$ equivalent of the PDE in [13], allowing efficient generation of the cm-rep models for 2D structures.

The cm-rep model is especially suitable for normalization of anatomical structures in multivariate datasets, because of its ability to extend boundary-based or skeleton-based correspondences to the interiors of structures [13 15]. For 2D objects, the cm-rep model establishes a shape-based coordinate system tailored to the object, where one coordinate (or two coordinates for 3D object) follows the skeleton, and the other coordinate goes from the skeleton to the boundary.

This paper presents an application where shape-based normalization of the MSCC is used to analyze patterns of commissural connectivity in the human brain as derived from diffusion tensor imaging. Shape-based normalization of the MSCC is compared to registration paradigms, with results favoring the shape-based approach.

\section{Method}

Sec 2.1 and Sec 2.2 briefly describe the two normalization techniques which will be compared in this paper, and Sec 2.3 introduces the method to validate the normalization technique under Diffusion MRI framework.

\subsection{Shape-Based Normalization}

There are many techniques in the shape analysis field that establish shape-based correspondences between boundaries of structures. The $\mathrm{cm}$-rep method has a unique property that allows boundary-based or skeleton-based correspondences to be propagated to the interiors of objects, thus enabling shape-based normalization.

The cm-rep method has been thoroughly explained in [13, 15]. The most important concept is medial axis, also called the Blum skeleton, which is defined as the locus of centers and radii of all the maximal inscribed disks (MIDs) inside of an object. The Process-Induced Symmetric Axis (PISA) [9] is closely related to the Blum skeleton. It is formed by the midpoints of the chords connecting the corresponding boundary points where the MID is tangent to the object's boundary.

The cm-rep models for anatomical structures can be constructed by fitting a deformable cm-rep template to binary segmentations of the structure. The interior of the $\mathrm{cm}$-rep model can be parameterized by a shape-based coordinate system where axis $t$ is the Process-Induced Symmetric Axis (PISA), and the other axis $\xi$ goes from the PISA to the boundary. Example of gridline of this coordinate system is illustrated in Fig.2(a). The normalization based on it is illustrated in Fig. 2(d). 
In this paper, equal arc length parameterization of the PISA is used to establish the correspondence on $t$ axis, and equal length parameterization of the line between PISA and the corresponding boundary is used to establish the correspondence on $\xi$ axis.

\subsection{Volumetric Registration Based Normalization}

Deformable registration is used to normalize the same dataset, and the results are compared with shape-based normalization. The symmetric diffeomorphic registration algorithm developed by Avants et al. [1], one of the state-of-the-art high-dimensional large deformation registration algorithms, is used in this paper.

The template used in the registration is constructed from the dataset itself, following the method described in [2]. Considering the homogeneity on the interior of MSCC, the segmented 2D mask image of MSCC is directly used for registration. And the DTIbased measurements are then warped to the template space according to the registration results.

\subsection{Validation of the Normalization}

Diffusion MRI studies provide an attractive framework within which to evaluate the performance of MSCC normalization via cm-reps and other techniques. With the help of diffusion tensor tractography, every location in the MSCC can be associated with a set of features derived from fiber tracts passing through that location. In a multi-subject experiment, these features can be used to detect structural differences between control and patient cohorts. The effect of different normalization methods on the statistical significance of detected differences can then be analyzed. Furthermore, normalization methods can be evaluated by examining how well they align anatomically labeled fiber tracts within cohorts. The following sections describe the approaches used to evaluate normalization from these different standpoints.

Features Derived from Diffusion Tensor Tractography. Diffusion tensor tractography is a tool for studying the white-matter connectivity in the brain. In our study we use two streamline tracking methods implemented in the open source Camino toolkit [3]. The FACT method, proposed by Mori et al [10], follows the local fiber orientation in each voxel, changing direction at voxel boundaries. We also track using a fixed, subvoxel step size $(0.4 \mathrm{~mm})$, following interpolated orientations taken from the vector field at each step using a simple eight-neighbor trilinear interpolation. We refer to this as the VINT (vector interpolation) method. Both of the tracking algorithms use an fractional anisotropy (FA) threshold and a curvature threshold to terminate the tracking. We repeat all our experiments for eight different tracking settings to check and ensure the stability of our result. The FA threshold is either 0.15 or 0.25 , the curvature threshold allows a maximum curvature of either 45 or 60 degrees over the length of a voxel, and the local fiber direction is determined by either the FACT or VINT algorithm.

The midsagittal plane is identified automatically according to the symmetry of the left and right hemisphere and the MSCC is manually segmented in the midsagittal plane. We then segment the fiber pathways of the MSCC in each subject by seeding streamline tractography in every voxel in the diffusion-tensor image and retaining only streamlines 
that intersect the MSCC. Following a similar method to Corouge et al [5], we examine the FA of diffusion along the length of each streamline. The FA of each voxel visited by a streamline is weighted by the length of the line segment in the voxel in order to compute a mean, giving each streamline an associated tract FA. The tract FA is plotted on the MSCC, as illustrated in Fig. 2(c). The value of each voxel is the mean tract FA of all streamlines that pass through that voxel.

MSCC Connectivity Labeling. A qualitative examination of the cortical connectivity is complimentary to the quantitative tract-based measures. Following [4, 7], we label the streamlines passing through the MSCC according to their cortical connectivity. To label the fibers, an anatomical atlas [14] is used, which divides each hemisphere of the cerebral cortex into four regions: frontal, parietal, temporal and occipital. The atlas is aligned to the T1-weighted image of each subject using a diffeomorphic image registration algorithm [1]. Examples of the warped atlas in space of the T1-weighted image are shown in Fig. 1. Then the warped atlas in the space of the T1-weighted image is further aligned to the space of the diffusion tensor image using the transformation that coregisters the T1-weighted image to the diffusion tensor image. Now each fiber derived from tractography in the diffusion image space can be assigned a label according to the cortical region of its endpoints. An example of labeled fibers is shown in Fig. 1. Finally, we can label each voxel in the MSCC according to the connectivity of fibers crossing it. If streamlines passing through the voxel connect to multiple cortical zones, we label the voxel according to the cortical zone that has the most fibers passing through the voxel. If no streamlines pass through a voxel, it has a clear label.

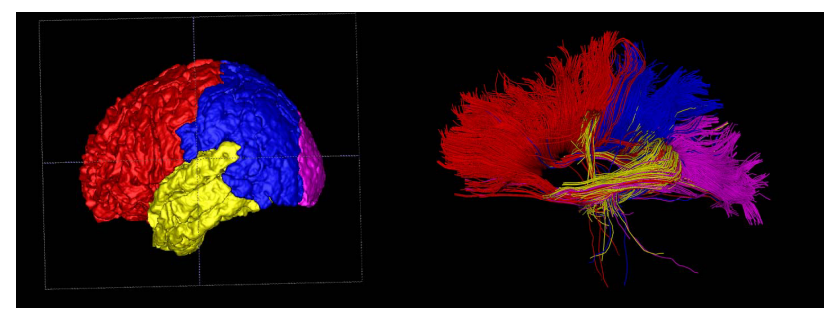

Fig. 1. Left: Examples of warped labeled atlas in the space of T1-weighted image; right: example of color labeled fibers. (Red for frontal lobes, blue for parietal lobes, yellow for temporal lobes and purple for occipital lobes).

\section{Results}

\subsection{Subjects and Data Acquisition}

Our evaluation experiments use a dataset from a chromosome $22 q 11.2$ deletion syndrome study. It includes 3.0-T high-resolution structural MRI and diffusion tensor MRI scans for 11 normally developing children and 19 children with the 22q11.2 syndrome. The structural MRI was acquired using a T1-weighted magnetization-prepared rapid gradient echo (MP-RAGE) sequence with the following scanning parameters: repetition 
time (TR) $1620 \mathrm{~ms}$, echo time (TE) $3.87 \mathrm{~ms}, 15^{\circ}$ flip angle, matrix size $=256 \times 192$, slice thickness of $1.0 \mathrm{~mm}$, spacing between slices of $1.0 \mathrm{~mm}$, yielding 160 axial slices with in-plane resolution of $0.98 \times 0.98 \mathrm{~mm}$. A single-shot, spin-echo, diffusion-weighted echo-planar imaging (EPI) sequence was used for the diffusion tensor MRI. The diffusion scheme was as follows: one image without diffusion gradients $\left(b=0 \mathrm{~s} / \mathrm{mm}^{2}\right)$, hereafter referred to as the $[b=0]$ image, followed by twelve images measured with twelve non-collinear and non-coplanar diffusion encoding directions isotropically distributed in space $\left(b=1000 \mathrm{~s} / \mathrm{mm}^{2}\right)$. Additional imaging parameters for the diffusion-weighted sequence were: $\mathrm{TR}=6500 \mathrm{~ms}$, TE $=99 \mathrm{~ms}, 90^{\circ}$ flip angle, number of averages $=6$, matrix size $=128 \times 128$, slice thickness $=3.0 \mathrm{~mm}$, spacing between slices $=3.0 \mathrm{~mm}$, 40 axial slices with in-plane resolution of $1.72 \times 1.72 \mathrm{~mm}$.

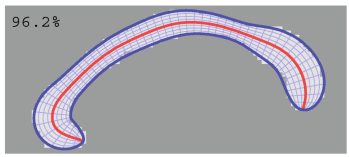

(a)

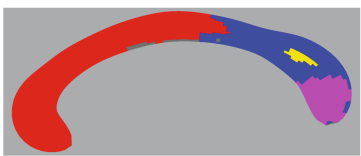

(b)

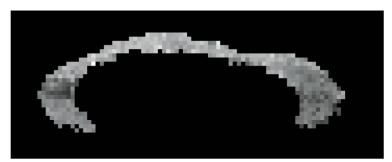

(c)

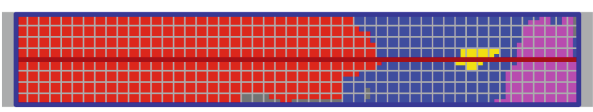

(d)

Fig. 2. (a)Illustration of PISA parameterization for one subject (Dice coefficient between the binary mask and its cm-rep model is given on the top). (b)The mean connectivity map obtained by cm-rep approach rendered on mean MSCC shape. (c) Example of tract FA plotted on the MSCC. (d) Illustration of shape-based normalization by plotting the mean connectivity map on shape-based coordinate system.

\subsection{Matching of the Connectivity Labels}

Given the connectivity-based labeling of the MSCC described in Sec. 2.3, it is possible to evaluate normalization algorithms based on how well they align connectivity labels across subjects. To do that, we measure the Dice overlap coefficient between normalized maps. Overlap is computed separately for each of the four labels in the connectivity map and is averaged among all pairs of subjects within each cohort. Table 1 lists the average overlaps for the FACT tracking method with parameters FA threshold 0.25 and curvature threshold 60 degrees, showing significantly higher overlap for the cmrep method. The comparison was repeated for the 8 variations of the tracking method, showing similar result. The maximum p-values among all 8 tracking methods are also included in Table 1 The difference in overlaps was statistically significant in almost all cases, with the exception of the temporal lobe (the temporal lobe occupies a very small portion of the connectivity map and is matched poorly by all normalization methods).

To help explaining the differences between the two normalization methods, we examine the Jacobian determinant maps associated with warping each subject into the normalized space. The average (over all 30 subjects in the study) variance of the Jacobian determinant inside MSCC is 0.22 for registration based normalization, and 0.06 
for cm-rep based normalization. As shown in Fig. 3, the Jacobian map of cm-rep normalization is much more uniform than that of registration. This is to be expected, since $\mathrm{cm}$-rep correspondences are more global in nature than correspondences based on local regularization priors, which are employed in registration. This difference in deformation fields can help explain better alignment of connectivity maps by the cm-rep method.

Table 1. Comparison of deformable registration based normalization and $\mathrm{cm}$-rep based normalization for matching the connectivity label of each lobe. The quality of label matching is quantified using Dice similarity coefficients between pairs of subjects. Statistics are carried out to measure the significance of the differences between normalization methods.

\begin{tabular}{|r||r||r|c|c|c|}
\hline & & FRONT & PAR & TEMP & OCC \\
\hline \hline CTRL(11 subjects) & registration & 0.735 & 0.481 & 0.124 & 0.514 \\
\hline & cm-rep & 0.811 & 0.553 & 0.134 & 0.633 \\
\hline & p-value & $<0.001$ & 0.006 & 0.387 & $<0.001$ \\
\hline & Max p-value among 8 methods & $<0.001$ & 0.030 & 0.478 & 0.007 \\
\hline 22Q PAT(19 subjects) & registration & 0.768 & 0.481 & 0.264 & 0.495 \\
\hline & cm-rep & 0.835 & 0.546 & 0.272 & 0.608 \\
\hline & p-value & $<0.001$ & $<0.001$ & 0.362 & $<0.001$ \\
\hline & Max p-value among 8 methods & $<0.001$ & $<0.001$ & 0.408 & $<0.001$ \\
\hline \hline All(30 subjects) & registration & 0.761 & 0.483 & 0.209 & 0.503 \\
\hline & cm-rep & 0.824 & 0.543 & 0.215 & 0.614 \\
\hline & p-value & $<0.001$ & $<0.001$ & 0.334 & $<0.001$ \\
\hline & Max p-value among 8 methods & $<0.001$ & $<0.001$ & 0.420 & $<0.001$ \\
\hline \hline
\end{tabular}
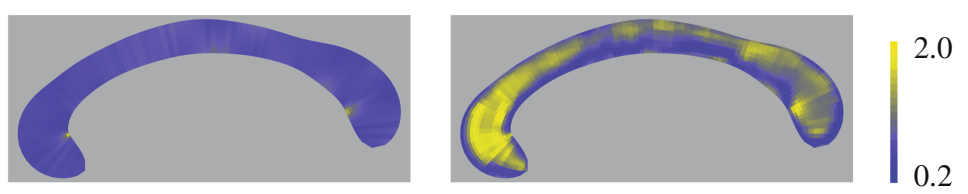

Fig. 3. Color encoded Jacobian determinant maps inside MSCC for one subject. Left: cm-rep mapping; right: deformable registration.

\subsection{Statistics on Tract FA Maps}

Finally, we compare the effect of normalization on the power of statistical analysis involving mean FA features derived from diffusion tensor tractography. As described in Sec. 2.3. a tract-wise mean FA value is associated with each point in the MSCC of each subject. Using each normalization method, the tract-wise FA maps are warped to a common template space, in which point-wise statistical analysis (a two-sample unpaired t-test comparing patients and controls) is performed. Regions of statistical significance in the template space are found using cluster analysis with permutation testing. Pixels with t-value higher than 3.13 is selected to build clusters, and permutation testing is used to build an empirical distribution of cluster size under the null hypothesis. For 
normalization based on region-of-interest registration, no clusters with $p$-value below 0.05 are detected, regardless of the tracking method used. In contrast, cm-rep normalization finds consistent results across 8 tracking method. Fig. 4 summarized the result for FACT tracking with a FA threshold of 0.25 and a curvature threshold of 60 degrees. A significant cluster in the mid-section of the MSCC is detected. We also collapsed the tract FA data on the PISA skeleton by summarizing the value of all points with the same $t$ PISA coordinate, and plot the summarized value along PISA skeleton in Fig. 4 The summarized tract FA values for control groups appear to have three peaks along the PISA skeleton, while the patient group does not have an obvious middle peak. The statistical analysis confirms the significance of this difference.
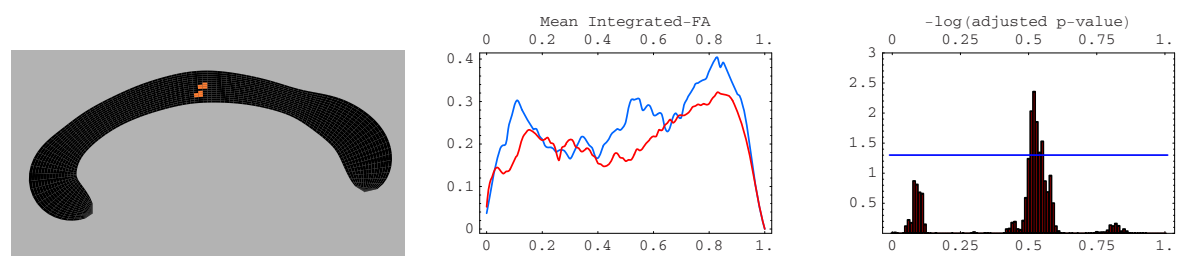

Fig. 4. This figure illustrates the differences of tract FA between the control group and 22q group, obtained using shape-based normalization. (1)Left: result of cluster analysis, areas with significant differences are shown in color. (2)Middle: the mean tract FA map of each groups after collapsing onto the PISA skeleton; the blue curves are for control group and red curves are for patient group. (3)Right: plot of -Log(adjusted p-values) for multiple statistical tests on the difference between the two groups after collapsing to skeleton; the p-values are corrected for multiple comparison using step down permutation; and the blue line is the significant threshold corresponding to adjusted- $\mathrm{p}=0.05$.

\section{Conclusion}

We have evaluated shape-based normalization method on MSCC in the context of a DTI study for 22q11.2 deletion syndrome. Results show that shape-based normalization aligns subregions of the MSCC, defined by connectivity, more accurately, and also helps increase the statistical power of group analysis in an experiment where tract FA is compared between two cohorts. These results suggest that cm-rep can be a more appropriate tool than the more widely used registration for normalizing the corpus callosum in white matter studies.

\section{References}

1. Avants, B.B., Gee, J.C.: Symmetric geodesic shape averaging and shape interpolation. In: ECCV Workshops CVAMIA and MMBIA, pp. 99-110 (2004)

2. Avants, B.B., Grossman, M., Gee, J.C.: Symmetric diffeomorphic image registration: Evaluating automated labeling of elderly and neurodegenerative cortex and hippocampus. In: Pluim, J.P.W., Likar, B., Gerritsen, F.A. (eds.) WBIR 2006. LNCS, vol. 4057, Springer, Heidelberg (2006) 
3. Cook, P.A., Bai, Y., Hall, M.G., Nedjati-Gilani, S., Seunarine, K.K., Parker, G.J.M., Alexander, D.C.: Camino: Open-source diffusion-MRI reconstruction and processing. In: Proceedings of the Scientific Meeting of the International Society for Magnetic Resonance in Medicine, p. 2759 (2006)

4. Cook, P.A., Zhang, H., Avants, B.B., Yushkevich, P.A., Alexander, D.C., Gee, J.C., Ciccarelli, O., Thompson, A.J.: An automated approach to connectivity-based partitioning of brain structures. In: Duncan, J.S., Gerig, G. (eds.) MICCAI 2005. LNCS, vol. 3749, pp. 164-171. Springer, Heidelberg (2005)

5. Corouge, I., Fletcher, P.T., Joshi, S., Gouttard, S., Gerig, G.: Fiber tract-oriented statistics for quantitative diffusion tensor MRI analysis. Medical Image Analysis 10, 786-798 (2006)

6. Davies, R.H., Twining, C.J., Cootes, T.F., Waterton, J.C., Taylor, C.J.: A minimum description length approach to statistical shape modeling. IEEE Trans. Med. Imaging 21(5), 525537 (2002)

7. Huang, H., Zhang, J., Jiang, H., Wakanaa, S., Poetschera, L., Miller, M.I., van Zijl, P.C.M., Hillis, A.E., Wytik, R., Mori, S.: DTI tractography based parcellation of white matter: Application to the mid-sagittal morphology of corpus callosum. neuroim 26, 195-205 (2005)

8. Kotcheff, A.C.W, Taylor, C.J.: Automatic construction of eigenshape models by genetic algorithm. Information Processing in Medical Imaging, 1-14 (1997)

9. Leyton, M.: Symmetry-curvature duality. Computer Vision, Graphics, and Image Processing 37(3), 327-341 (1987)

10. Mori, S., Crain, B.J., Chacko, V.P., van Zijl, P.C.M.: Three dimensional tracking of axonal projections in the brain by magnetic resonance imaging. Ann. Neurol. 45, 265-269 (1999)

11. Pizer, S.M., Fletcher, P.T., Joshi, S., Thall, A., Chen, J.Z., Fridman, Y., Fritsch, D.S., Gash, A.G., Glotzer, J.M., Jiroutek, M.R., Lu, C., Muller, K.E., Tracton, G., Yushkevich, P., Chaney, E.L.: Deformable m-reps for 3D medical image segmentation. International Journal of Computer Vision 55(2), 85-106 (2003)

12. Rohlfing, T.: Transformation model and constraints cause bias in statistics on deformation fields. In: Larsen, R., Nielsen, M., Sporring, J. (eds.) MICCAI 2006(1). LNCS, vol. 4190, pp. 207-214. Springer, Heidelberg (2006)

13. Sun, H., Yushkevich, P.A., Zhang, H., Gee, J.C., Simon, T.J.: Efficient generation of shapebased reference frames for the corpus callosum for dti-based connectivity analysis. In: CVPRW 2006. Proceedings of the 2006 Conference on Computer Vision and Pattern Recognition Workshop, p. 87. IEEE Computer Society, Washington (2006)

14. Yushkevich, P., Dubb, A., Xie, Z., Gur, R., Gur, R., Gee, J.: Regional structural characterization of the brain of schizophrenia patients. Acad. Radiol. 12(10), 1250-1261 (2005)

15. Yushkevich, P.A., Zhang, H., Gee, J.: Continuous medial representation for anatomical structures. IEEE Trans. Med. Imaging (to appear)

16. Yushkevich, P.A., Zhang, H., Gee, J.C.: Statistical modeling of shape and appearance using the continuous medial representation. In: MICCAI. Medical Image Computing and Computer-Assisted Intervention, vol. 2, pp. 725-732 (2005) 